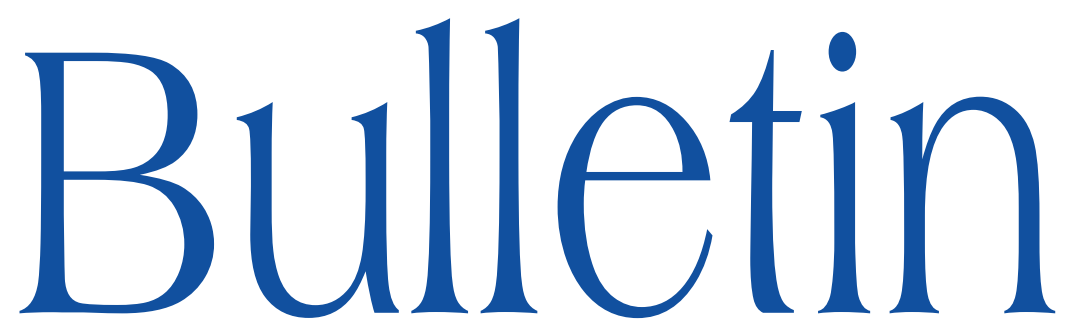

de la SOCIÉTÉ MATHÉMATIQUE DE FRANCE

\title{
COLEFF-HERRERA CURRENTS, DUALITY AND NOETHERIAN OPERATORS
}

\author{
Mats Andersson
}

\section{Tome 139}

Fascicule 4

2011 


\title{
COLEFF-HERRERA CURRENTS, DUALITY, AND NOETHERIAN OPERATORS
}

\author{
BY MATS ANDERSSON
}

\begin{abstract}
Let $I$ be a coherent subsheaf of a locally free sheaf $\vartheta\left(E_{0}\right)$ and suppose that $\mathcal{F}=\theta\left(E_{0}\right) / \mathcal{I}$ has pure codimension. Starting with a residue current $R$ obtained from a locally free resolution of $\mathcal{F}$ we construct a vector-valued Coleff-Herrera current $\mu$ with support on the variety associated to $\mathscr{F}$ such that $\phi$ is in $\mathscr{J}$ if and only if $\mu \phi=0$. Such a current $\mu$ can also be derived algebraically from a fundamental theorem of Roos about the bidualizing functor, and the relation between these two approaches is discussed. By a construction due to Björk one gets Noetherian operators for $\mathcal{J}$ from the current $\mu$. The current $R$ also provides an explicit realization of the Dickenstein-Sessa decomposition and other related canonical isomorphisms.

RÉsumé (Courants de Coleff-Herrera, dualité et opérateurs noethériens)

Soit $I$ un sous-faisceau cohérent d'un faisceau localement libre $\theta\left(E_{0}\right)$ et supposons que $\mathscr{F}=\vartheta\left(E_{0}\right) / \mathscr{I}$ ait une codimension pure. En partant d'un courant résiduel $R$, obtenu à partir d'une résolution localement libre de $\mathcal{F}$, nous construisons un courant de Coleff-Herrera vectoriel $\mu$ à support sur la variété associée à $\mathscr{F}$, tel que $\phi$ soit dans I si et seulement si $\mu \phi=0$. Un tel courant $\mu$ peut également être dérivé algébriquement grâce à un théorème fondamental de Roos sur le foncteur bidualisant, et nous étudions le lien entre les deux approches. Par une construction due à Björk, on obtient des opérateurs noethériens pour $I$ à partir du courant $\mu$. Le courant $R$ nous fournit également une réalisation explicite de la décomposition de Dickenstein-Sessa, ainsi que d'autres isomorphismes canoniques afférents.
\end{abstract}

Texte reçu le 9 juillet 2009, révisé le 6 octobre 2010 et le 29 juin 2011.

Mats Andersson, Department of Mathematics, Chalmers University of Technology and the University of Gothenburg, S-41296 Göteborg, Sweden E-mail : matsa@math.chalmers.se

2000 Mathematics Subject Classification. - 32C30, 32A27.

Key words and phrases. - Coleff-Herrera current, duality, Noetherian operators, residue current.

The author was partially supported by the Swedish Natural Science Research Council. 


\section{Introduction}

A function $\phi$ in the local ring $\theta_{0}$ in one complex variable belongs to the ideal $I$ generated by $z^{m}$ if and only if

$$
\mathcal{L}_{\ell} \phi(0)=0, \ell=0, \ldots, m-1,
$$

where $\mathscr{L}_{\ell}=\partial^{\ell} / \partial z^{\ell}$. These conditions can be elegantly expressed by the single equation $\phi \bar{\partial}\left(1 / z^{m}\right)=0$, where $1 / z^{m}$ is the usual principal value distribution. Moreover, the current $\mu=\bar{\partial}\left(1 / z^{m}\right)$ is canonical up to a non-vanishing holomorphic factor. There is a well-known multivariable generalization. Let $f=\left(f^{1}, \ldots, f^{p}\right)$ be a tuple of holomorphic functions in a neighborhood of the origin in $\mathbb{C}^{n}$ that defines a complete intersection, i.e., the codimension of $Z^{f}=\{f=0\}$ is equal to $p$. Then the Coleff-Herrera product

$$
\mu^{f}=\bar{\partial} \frac{1}{f^{1}} \wedge \cdots \wedge \bar{\partial} \frac{1}{f^{p}},
$$

introduced in [9], is a $\bar{\partial}$-closed $(0, p)$-current with support on $Z^{f}$, and it is independent (up to a nonvanishing holomorphic factor) of the choice of generators of the ideal sheaf $I$ generated by $f$. It was proved in [10] and [17] that $I$ coincides with the ideal sheaf ann $\mu^{f}$ of holomorphic functions $\phi$ such that the current $\mu^{f} \phi$ vanishes. This is often referred to as the duality principle.

The Coleff-Herrera product is the model for a general Coleff-Herrera current introduced by Björk: Given a variety $Z$ of pure codimension $p$ we say that a (possibly vector-valued) $(0, p)$-current $\mu$ (with support on $Z$ ) is a ColeffHerrera current on $Z, \mu \in \mathscr{C H} \mathscr{H}_{Z}$, if it is $\bar{\partial}$-closed, annihilated by $\bar{J}_{Z}$ (i.e., $\bar{\xi} \mu=0$ for each holomorphic $\xi$ that vanishes on $Z$ ), and has the standard extension property SEP. This means, roughly speaking, that $\mu$ has no "mass" concentrated on any subvariety of higher codimension; in particular that $\mu$ is determined by its values on $Z_{r e g}$, see, e.g., [7] or [3], and Section 2.1. The SEP implies that ann $\mu$ has pure dimension, see, e.g., Proposition 5.3 in [5]. The condition $\bar{g}_{Z} \mu=0$ means that $\mu$ only involves holomorphic derivatives. Following Björk, see [7], one can quite easily find a finite number of holomorphic differential operators $\mathscr{L}_{\ell}$ such that $\phi \mu=0$ if and only if $\mathscr{L}_{1} \phi=\cdots=\mathscr{L}_{\nu} \phi=0$ on $Z$; i.e., a (complete) set of Noetherian operators for ann $\mu$. In this paper we use the residue theory developed in [4] and [5] to extend the duality for a complete intersection to a general pure-dimensional ideal (or submodule of a locally free) sheaf. In particular we can express such an ideal as the annihilator of a finite set of Coleff-Herrera currents (Theorem 1.2 and its corollaries). JanErik Björk has pointed out to us that one can deduce the same duality result from a fundamental theorem of Jan-Erik Roos, [18], about purity for a module in terms of the bidualizing sheaves, combined with some other known facts that will be described below. However our approach gives a representation of the 
duality and the Coleff-Herrera currents in terms of one basic residue current, that we first describe.

To begin with, let $I$ be any coherent subsheaf of a locally free sheaf $\theta\left(E_{0}\right)$ over a complex manifold $X$, and assume that

$$
0 \rightarrow \Theta\left(E_{N}\right) \stackrel{f_{N}}{\longrightarrow} \cdots \stackrel{f_{3}}{\longrightarrow} \Theta\left(E_{2}\right) \stackrel{f_{2}}{\longrightarrow} \Theta\left(E_{1}\right) \stackrel{f_{1}}{\longrightarrow} \Theta\left(E_{0}\right)
$$

is a locally free resolution of $\mathcal{F}=\Theta\left(E_{0}\right) / \mathcal{I}$. Here $\Theta\left(E_{k}\right)$ denotes the locally free sheaf associated to the vector bundle $E_{k}$ over $X$. If $X$ is Stein, then one can find such a resolution in a neighborhood of any given compact subset. We will assume that $\mathcal{F}$ has codimension $p>0$; cf., Remark 2. Then $f_{1}$ is (can be assumed to be) generically surjective, and the analytic set $Z$ where it is not surjective has codimension $p$ and coincides with the zero set of the ideal sheaf ann $\mathcal{F}$. In [4] we defined, given Hermitian metrics on $E_{k}$, a residue current $R=R_{p}+R_{p+1}+\cdots$ with support on $Z$, where $R_{k}$ is a $(0, k)$-current that takes values in $\operatorname{Hom}\left(E_{0}, E_{k}\right)$, such that a holomorphic section $\phi \in \Theta\left(E_{0}\right)$ is in $\checkmark$ if and only if $R \phi=0$.

Recall that $\mathcal{F}$ has pure codimension $p$ if the associated prime ideals (of each stalk) all have codimension $p$. The starting point in this paper is the following result that follows from [5] (see also Section 7 below); as we will see later on it is in a way equivalent to Roos' characterization of purity.

THEOREM 1.1. - The sheaf $\mathcal{F}=\Theta\left(E_{0}\right) / \mathcal{I}$ has pure codimension $p$ if and only if $I$ is equal to the annihilator of $R_{p}$, i.e.,

$$
I=\left\{\phi \in \theta\left(E_{0}\right) ; R_{p} \phi=0\right\} \text {. }
$$

If $\mathcal{F}$ is Cohen-Macaulay we can choose a resolution (1.1) with $N=p$, and then $R=R_{p}$ is a matrix of $\mathscr{C} \mathscr{H}_{Z}$-currents which thus solves our problem. However, in general $R_{p}$ is not $\bar{\partial}$-closed even if $\mathcal{F}$ has pure codimension. Let

$$
0 \rightarrow \Theta\left(E_{0}^{*}\right) \stackrel{f_{1}^{*}}{\longrightarrow} \Theta\left(E_{1}^{*}\right) \stackrel{f_{2}^{*}}{\longrightarrow} \cdots \stackrel{f_{p-1}^{*}}{\longrightarrow} \Theta\left(E_{p-1}^{*}\right) \stackrel{f_{p}^{*}}{\longrightarrow} \Theta\left(E_{p}^{*}\right) \stackrel{f_{p+1}^{*}}{\longrightarrow}
$$

be the dual complex of (1.1) and let

$$
\mathcal{H}^{k}\left(\theta\left(E_{\bullet}^{*}\right)\right)=\frac{\operatorname{Ker}_{f_{k+1}^{*}} \theta\left(E_{k}^{*}\right)}{f_{k}^{*} \Theta\left(E_{k-1}^{*}\right)}
$$

be the associated cohomology sheaves. It turns out that for each choice of $\xi \in \Theta\left(E_{p}^{*}\right)$ such that $f_{p+1}^{*} \xi=0$, the current $\xi R_{p}$ is in $\mathscr{C H}_{Z}\left(E_{0}^{*}\right)$, and we have in fact a bilinear (over $\Theta$ ) pairing

$$
\mathcal{H}^{p}\left(\Theta\left(E_{\bullet}^{*}\right)\right) \times \mathcal{F} \rightarrow \mathscr{C H}{ }_{Z}, \quad(\xi, \phi) \mapsto \xi R_{p} \phi .
$$

Moreover, (1.4) is independent of the choice of Hermitian metrics on $E_{k}$. It is well-known that the sheaves in (1.3) represent the intrinsic sheaves $\varepsilon_{x} t_{\theta}^{k}(\mathcal{F}, \Theta)$. (If $Z$ does not have pure codimension $p$ then we define $\mathscr{G} \mathcal{H}_{Z}$ as $\mathscr{C} \mathcal{H}_{Z^{\prime}}$, where 
$Z^{\prime}$ is the union of irreducible components of codimension $p$; this is reasonable, in view of the SEP.)

TheOREM 1.2. - Assume that $\mathcal{F}$ has codimension $p>0$. The pairing (1.4) induces an intrinsic pairing

$$
\mathcal{E} x t_{\theta}^{p}(\mathcal{F}, \theta) \times \mathcal{F} \rightarrow \mathscr{C H}_{Z}
$$

If $\mathcal{F}$ has pure codimension, then the pairing is non-degenerate.

Notice that $\mathscr{H a m}(\mathcal{F}, \mathscr{C H} Z)$ is the subsheaf of $\mathscr{H} \operatorname{Cam}\left(\theta\left(E_{0}\right), \mathscr{C H} Z\right)=\mathscr{C H} \mathcal{H}_{Z}\left(E_{0}^{*}\right)$ consisting of all Coleff-Herrera currents $\mu$ with values in $E_{0}^{*}$ such that $\mu \phi=0$ for all $\phi \in \mathcal{J}$. It follows that we have the equality

$$
I=\left\{\phi \in \Theta\left(E_{0}\right) ; \mu \phi=0 \text { for all } \mu \in \mathcal{H} \operatorname{tam}(\mathcal{F}, \mathscr{C H} Z)\right\}
$$

if $\mathcal{F}$ is pure. The sheaf $\mathcal{H}^{p}\left(\mathscr{\theta}\left(E_{\bullet}^{*}\right)\right)$ is coherent and thus locally finitely generated. Therefore we have now a solution to our problem:

Corollary 1.3. - Assume that $\mathcal{F}$ has pure codimension. If $\xi_{1}, \ldots, \xi_{\nu} \in$ $\Theta\left(E_{p}^{*}\right)$ generate $\mathcal{H}^{p}\left(\Theta\left(E_{\bullet}^{*}\right)\right)$, then $\mu_{j}=\xi_{j} R_{p}$ are in $\mathcal{H} \operatorname{tam}\left(\mathcal{F}, \mathscr{C H}_{Z}\right)$ and

$$
\text { I }=\cap_{j=1}^{\nu} \text { ann } \mu_{j} \text {. }
$$

REMARK 1. - If $I$ is not pure, one obtains a decomposition (1.7) after a preliminary decomposition $\mathscr{I}=\cap \mathcal{I}_{\nu}$, where each $\mathcal{I}_{\nu}$ has pure codimension.

In case of a complete intersection, $\mathscr{E} x t^{p}(\mathcal{F}, \theta)$ is isomorphic to $\mathcal{F}$ itself. If $\mathcal{F}=\Theta\left(E_{0}\right) / \mathcal{I}$ is a sheaf of Cohen-Macaulay modules there is also a certain symmetry: If (1.1) is a resolution with $N=p$, then it is well-known, cf., also Example 4 below, that the dual complex (1.2) is a resolution of $\theta\left(E_{p}^{*}\right) / \mathcal{I}^{*}$, where $\mathcal{I}^{*}=f_{p}^{*} \theta\left(E_{p-1}^{*}\right) \subset \vartheta\left(E_{p}^{*}\right)$, and we have

Corollary 1.4. - If $\Theta\left(E_{0}\right) / \mathcal{I}$ is Cohen-Macaulay, then $\Theta\left(E_{p}^{*}\right) / \mathscr{I}^{*}$ is Cohen-Macaulay as well and we have a non-degenerate pairing

$$
\theta\left(E_{0}\right) / \mathscr{I} \times \Theta\left(E_{p}^{*}\right) / I^{*} \rightarrow \mathscr{C H} Z, \quad(\xi, \phi) \mapsto \xi R_{p} \phi
$$

REMARK 2. - Assume that $\mathcal{F}$ has codimension $p=0$, or equivalently, ann $\mathcal{F}=0$. If it is pure, i.e., $(0)$ is the only associated prime ideal, then there is a homomorphism $f_{0}: \Theta\left(E_{0}\right) \rightarrow \Theta\left(E_{-1}\right)$ such that $\mathscr{I}=\operatorname{Ker} f_{0}$. It is natural to consider $f_{0}$ as a Coleff-Herrera current $\mu$ associated with the zero-codimensional "variety" $X$. Then $\mathscr{I}=$ ann $\mu$ and thus analogues of Theorem 1.1 and Corollary 1.3 still hold. 
The duality discussed here leads to a generalization of the Dickenstein-Sessa decomposition that we now will describe. It was proved by Malgrange, see, e.g., [7], that the analytic sheaf of distributions $\mathscr{C}$ is stalkwise injective. Thus the double complex

$$
\operatorname{Ham}_{\vartheta}\left(\theta\left(E_{\ell}\right), \mathscr{C}^{0, k}\right)=\mathscr{C}^{0, k}\left(E_{\ell}^{*}\right)
$$

with differentials $\bar{\partial}$ and $f^{*}$, is exact except at $k=0$ and $\ell=0$, where we have the cohomology sheaves $\Theta\left(E_{\ell}^{*}\right)$ and $\operatorname{Ham}\left(\mathcal{F}, \mathscr{C}^{0, \bullet}\right)$, respectively. By standard homological algebra, we therefore have natural isomorphisms

$$
\mathcal{H}^{k}\left(\mathscr{\theta}\left(E_{\bullet}^{*}\right)\right) \simeq \mathcal{H}^{k}\left(\mathcal{H a m}\left(\mathcal{F}, \mathscr{C}^{0, \bullet}\right)\right) .
$$

The residue calculus also gives

TheOrem 1.5. - Assume that $\operatorname{codim} \mathcal{F}=p>0$. Both mappings

$$
\mathcal{H}^{p}\left(\theta\left(E_{\bullet}^{*}\right)\right) \stackrel{\Psi}{\simeq} \operatorname{Ham}\left(\mathcal{F}, \mathscr{G} \mathcal{H}_{Z}\right) \simeq \mathcal{H}^{p}\left(\mathcal{H} \operatorname{tam}\left(\mathcal{F}, \mathscr{C}^{0, \bullet}\right)\right)
$$

are isomorphisms, and the composed mapping coincides with the isomorphism (1.9).

These isomorphisms seem to be known as "folklore" since long ago, cf., Section 4 below. Our contribution should be the proof by residue calculus, and especially, the realization of the mapping $\Psi$ as $\xi \mapsto \xi R_{p}$.

ExAmPLE 1. - If $\mu \in \mathscr{C H} \mathcal{H}_{Z}$ is annihilated by $I$ it follows that we have the factorization $\mu=\xi R_{p}$. There are analogous isomorphisms where $\theta$ is replaced by $\Omega^{r}$, the sheaf of holomorphic $(r, 0)$-forms, and Coleff-Herrera currents of bidegree $(r, p), \mathscr{C} \mathcal{H}_{Z}^{r}=\mathscr{C} \mathcal{H}_{Z} \otimes_{\vartheta} \Omega^{r}$. For instance it follows that there is a factorization

$$
[Z]=\xi R_{p},
$$

where $[Z]$ is the Lelong current, and $\xi$ is in $\Omega^{p}\left(E_{p}^{*}\right)$ with $f_{p+1}^{*} \xi=0$.

EXAMPLE 2. - We can rephrase the second isomorphism in (1.10) as the decomposition

$$
\begin{aligned}
\operatorname{Ker}\left(\operatorname{Ham}\left(\mathcal{F}, \mathscr{C}^{0, p}\right) \stackrel{\bar{\partial}}{\rightarrow}\left(\operatorname{Ham}\left(\mathcal{F}, \mathscr{C}^{0, p+1}\right)\right)=\right. \\
\quad=\operatorname{Ham}\left(\mathcal{F}, \mathscr{H} \mathcal{H}_{Z}\right) \oplus \bar{\partial} \operatorname{Ham}\left(\mathcal{F}, \mathscr{C}^{0, p-1}\right) .
\end{aligned}
$$

For a given $\bar{\partial}$-closed $(0, p)$-current $\mu$ (with values in $E_{0}^{*}$ and annihilated by $\left.\Im\right)$, its canonical projection in $\operatorname{Ham}\left(\mathcal{F}, \mathscr{C H}_{Z}\right)$ is given by $\xi R_{p}$, where $\xi$ is obtained from $\mu$ via the isomorphism (1.9). 
ExAmple 3. - Assume that $Z$ has pure codimension $p$ and let $\mathscr{C}_{Z}^{0, k}$ denote the sheaf of $(0, k)$-currents with support on $Z$. If $\mathcal{F}$ has support on $Z$, then $\operatorname{Ham}\left(\mathcal{F}, \mathscr{C}^{0, k}\right)=\operatorname{Ham}\left(\mathcal{F}, \mathscr{C}_{Z}^{0, k}\right)$. Since any current with support on $Z$ must be annihilated by some power of $\mathscr{I}_{Z},(1.11)$ implies the decomposition

$$
\operatorname{Ker}\left(\mathscr{C}_{Z}^{0, p} \stackrel{\bar{\partial}}{\rightarrow} \mathscr{C}_{Z}^{0, p+1}\right)=\mathscr{C H}_{Z} \oplus \bar{\partial} \mathscr{C}_{Z}^{0, p-1}
$$

that was first proved in [10] by Dickenstein and Sessa (in the case of a complete intersection; see [7] for the general case).

The main results are proved in Section 3. In Section 4 we sketch a purely algebraic proof of Theorem 1.2 (except for the explicit residue representation) based on Roos' theorem. In Section 5 we consider in some more detail the absolute case, i.e., $p=n$, and in Section 6 we briefly discuss a cohomological variant of the duality. In Section 7 we consider a partial generalization of (1.10) to $k>p$; again we can trace residue manifestations of Roos' theorem.

In Section 2 we collect some basic material about residue currents. For the reader's convenience we include Björk's construction of Noetherian operators for the ideal ann $\mu$. To further exemplify the utility of the residue calculus, we include a proof of Malgrange's theorem by means of residues and integral formulas in Section 2.3.

All results above have natural analogues for polynomial ideals and modules: Let $I$ be a submodule of $\mathbb{C}\left[z_{1}, \ldots, z_{n}\right]^{r}$, and assume that $F=\mathbb{C}\left[z_{1}, \ldots, z_{n}\right]^{r} / I$ has positive codimension $p$. From a free resolution of the of the corresponding homogeneous module over the graded ring $\mathbb{C}\left[z_{0}, \ldots, z_{n}\right]$ we constructed in [4] a residue current on $\mathbb{P}^{n}$ whose restriction $R$ to $\mathbb{C}^{n}$ has the property that $\Phi \in$ $\mathbb{C}\left[z_{1}, \ldots, z_{n}\right]^{r}$ is in $I$ if and only if $R \Phi=0$ in $\mathbb{C}^{n}$. If $F$ has pure codimension, then precisely as in the semi-global case above we have that $\Phi$ is in $I$ if and only if $R_{p} \Phi=0$. By the same proofs we get complete analogues of Theorem 1.2 and its corollaries. In particular if $F$ is pure, we get a finite number of global Coleff-Herrera currents $\mu_{j}=\xi R_{p}$ such that $\Phi \in I$ if and only if $\mu_{j} \Phi=0$ for each $j$. Moreover, since $R_{p}$ has a current extension to $\mathbb{P}^{n}$, following the proof in Section 2.1 with $\Omega=\mathbb{C}^{n}$, we obtain for each $\mu_{j}$ a finite set of differential operators $\mathscr{L}_{j \ell}$ with polynomial coefficients such that $\mathscr{L}_{j \ell} \Phi$ vanishes on $Z$ for all $\ell$ if and only if $\Phi$ is in the annihilator of $\mu_{j}$. Starting with a primary decomposition of $I$ we obtain in this way a complete proof of the existence of Noetherian operators for an arbitrary polynomial ideal, a fact first proved by Ehrenpreis and Palamodov as the corner stone in the celebrated fundamental principle, see [11], [16], [13], and [6]. For a discussion about effectivity, see [15]. 
Acknowledgements. - I am grateful to Jan-Erik Björk for invaluable discussions on these matters, and for communicating the arguments in Section 4. I also would like to thank the referee for important suggestions.

\section{Some elements of residue theory}

Let $X$ be an $n$-dimensional complex manifold. In [5] was introduced the sheaf of pseudomeromorphic currents $\mathscr{P} M$. Roughly speaking a current $\mu$ is pseudomeromorphic if locally it is the push-forward under a modification $\pi: \tilde{X} \rightarrow X$ of a (finite sum of) currents like

$$
\bar{\partial} \frac{1}{s_{1}^{\alpha_{1}}} \wedge \cdots \wedge \bar{\partial} \frac{1}{s_{q}^{\alpha_{q}}} \wedge \frac{\omega}{s_{q+1}^{\alpha_{q+1}} \cdots s_{n}^{\alpha_{n}}},
$$

where $s$ is a local coordinate system and $\omega$ is a smooth form with compact support. Here $q$ may be 0 which means that we have no residue factor but only principal value factors. The sheaf $\mathscr{P S M}$ is closed under $\bar{\partial}$ and multiplication with smooth forms. It turns out that if $\mu$ is in $\mathscr{P} M$ and $V$ is a subvariety, then the restriction of $\mu$ to the open set $X \backslash V$ has a natural extension to a pseudomeromorphic current $\mathbf{1}_{X \backslash V} \mu$ on $X$ such that $\mathbf{1}_{V} \mu:=\mu-\mathbf{1}_{X \backslash V} \mu$ has support on $V$. If $h$ is any holomorphic tuple such that $V=\{h=0\}$, then $\lambda \mapsto|h|^{2 \lambda} \mu$, that is well-defined if $\operatorname{Re} \lambda \gg 0$, has a current-valued analytic continuation to $\operatorname{Re} \lambda>-\epsilon$, and the value at $\lambda=0,\left.|h|^{2 \lambda} \mu\right|_{\lambda=0}$, is precisely $\mathbf{1}_{X \backslash V} \mu$.

If $\mu$ is in $\mathscr{P M}$ and has support on $V$, then $\bar{I}_{V} \mu=0$, i.e., $\bar{\xi} \mu=0$ for each holomorphic function that vanishes on $V$. If $\mu$ has support on $V$ we say that it has the standard extension property, SEP, if $\mathbf{1}_{W} \mu=0$ for each $W \subset V$ of positive codimension. For (the equivalence to) the more classical way to define SEP, see [3] Proposition 5.1. We also have the dimension principle:

Proposition 2.1 ([5], Corollary 2.4). - If $\mu \in \mathscr{P M}$ has bidegree $(r, k)$ and support on a variety $V$ of codimension $>k$, then $\mu=0$.

It follows that if $\mu$ has bidegree $(r, p)$ and support on $V$ with codimension $p$ then it has automatically the SEP with respect to $V$.

2.1. Coleff-Herra currents and Noetherian operators. - Let $V$ be a subvariety with pure codimension $p$. We define the sheaf of Coleff-Herra currents $\mathscr{C} \mathcal{H}_{V}^{r}$ as the subsheaf of $\mathscr{P} M$ of currents of bidegree $(r, p)$ that has support on $V$ and are $\bar{\partial}$-closed. See Proposition 5.1 in [3] for an equivalent definition. 
THEOREM 2.2 (Björk [7]). — Let $V$ be a germ of an analytic variety of pure codimension $p$ at $0 \in \mathbb{C}^{n}$. There is a neighborhood $\Omega$ of 0 such that for each $\mu \in \mathscr{G} \mathcal{H}_{V}\left(E_{0}^{*}\right)$ in $\Omega$, there are holomorphic differential operators $\mathscr{L}_{1}, \ldots, \mathscr{L}_{\nu}$ in $\Omega$ such that for any $\phi \in \Theta\left(E_{0}\right), \mu \phi=0$ if and only if

$$
\mathscr{L}_{1} \phi=\cdots=\mathscr{L}_{\nu} \phi=0 \text { on } V \text {. }
$$

Proof. - In a suitable pseudoconvex neighhborhood $\Omega$ of 0 we can find holomorphic functions $f_{1}, \ldots, f_{p}$, forming a complete intersection, such that $V \cap \Omega$, henceforth denoted just $V$, is a union of irreducible components of $V_{f}=\{f=$ $0\}$, and such that $d f_{1} \wedge \cdots \wedge d f_{p} \neq 0$ on $V \backslash W$, where $W$ is a hypersurface not containing any component of $V_{f}$. By a suitable choice of coordinates $(\zeta, \omega) \in \mathbb{C}^{n-p} \times \mathbb{C}^{p}$ we may assume that $W$ is the zero set of $h=\operatorname{det}(\partial f / \partial \omega)$. Let $z=\zeta, w=f(\zeta, \omega)$. Since $d(z, w) / d(\zeta, \omega)=h$, locally in $\Omega \backslash W,(z, w)$ is a holomorphic coordinate system. If we take the multiindex $M$ so large that $\mu$ is annihilated by $f_{j}^{M_{j}+1}$, it follows from [7] (or Theorem 4.1 in [3]) that there is a holomorphic function $A \in \Omega$ such that

$$
\mu=A \bar{\partial} \frac{1}{f_{1}^{M_{1}+1}} \wedge \cdots \wedge \bar{\partial} \frac{1}{f_{p}^{M_{p}+1}}
$$

in $\Omega$. Thus locally in $\Omega \backslash W$,

$$
\mu . \xi=\int_{w=0} \sum_{\alpha \leq M} c_{\alpha} \frac{\partial^{M-\alpha} A(z, 0)}{\partial w^{M-\alpha}} \gamma \neg \frac{\partial^{\alpha} \xi}{\partial w^{\alpha}},
$$

where $\gamma \neg$ is contraction with the vector field

$$
\gamma=\frac{\partial}{\partial w_{p}} \wedge \cdots \wedge \frac{\partial}{\partial w_{1}} .
$$

Now $\mu \phi=0$ if and only if for all test forms $\xi$,

$$
0=\mu \phi . \xi=\int_{w=0} \sum_{\ell \leq M}\left(Q_{\ell} \phi\right) \gamma \neg \frac{\partial^{\ell}}{\partial w^{\ell}} \xi,
$$

where

$$
Q_{\ell}=\sum_{\ell \leq \alpha \leq M} c_{\alpha, \ell} \frac{\partial^{M-\alpha} A}{\partial w^{M-\alpha}} \frac{\partial^{\alpha-\ell}}{\partial w^{\alpha-\ell}} .
$$

Applying to $\xi=w^{\ell} \eta$ (induction over $\ell$ downwards) it follows that (2.2) holds for all $\xi$ if and only if $Q_{\ell} \phi=0$ (locally) on $V \backslash W$ for all $\ell \leq M$.

However, $\partial \omega / \partial w=(\partial f / \partial \omega)^{-1}=\gamma / h$ where $\gamma$ is a holomorphic matrix in $\Omega$. It follows that $\mathscr{L}_{\ell}=h^{N} Q_{\ell}$ are well-defined and holomorphic in $\Omega$ if $N$ is large enough, and by the SEP, $\mu \phi=0$ in $\Omega$ if and only if (2.1) holds. 
One also get a global (in $\Omega$ ) representation of $\mu$ in this way: Notice that for some $L, \tilde{\gamma}=h^{L} \gamma$ is holomorphic in $\Omega$. One can verify that (with $\mathscr{L}=\mathscr{L}_{0}$ ), actually

$$
\mu . \xi=\int_{Z} \frac{1}{h^{M+L}} \tilde{\gamma} \neg \mathscr{L} \xi
$$

for all test forms, if the right hand side is interpreted as a principal value.

2.2. Residue currents associated with Hermitian complexes. - We first have to recall the construction in [4]. Let

$$
0 \rightarrow E_{N} \stackrel{f_{N}}{\longrightarrow} \cdots \stackrel{f_{3}}{\longrightarrow} E_{2} \stackrel{f_{2}}{\longrightarrow} E_{1} \stackrel{f_{1}}{\longrightarrow} E_{0} \rightarrow \cdots \stackrel{f_{-M+1}}{\longrightarrow} E_{-M} \rightarrow 0
$$

be a generically exact complex of Hermitian vector bundles over a complex manifold $X$, and let

$$
0 \rightarrow \Theta\left(E_{N}\right) \stackrel{f_{N}}{\longrightarrow} \cdots \stackrel{f_{1}}{\longrightarrow} \Theta\left(E_{0}\right) \longrightarrow \cdots \stackrel{f_{-M+1}}{\longrightarrow} \Theta\left(E_{-M}\right) \rightarrow 0
$$

be the corresponding complex of locally free sheaves. Assume that (2.3) is pointwise exact outside the variety $Z$. Furthermore, over $X \backslash Z$ let $\sigma_{k}: E_{k-1} \rightarrow$ $E_{k}$ be the minimal inverses of $f_{k}$. Then $f \sigma+\sigma f=I_{E}$, where $I_{E}$ is the identity on $E=\oplus E_{k}, f=\oplus f_{k}$ and $\sigma=\oplus \sigma_{k}$. The bundle $E$ has a natural superbundle structure $E=E^{+} \oplus E^{-}$, where $E^{+}=\oplus E_{2 k}$ and $E^{-}=\oplus E_{2 k+1}$, and $f$ and $\sigma$ are odd mappings with respect to this structure, see, e.g., [4] for more details. The operator $\nabla=f-\bar{\partial}$ acts on $\mathscr{C}^{0, \bullet}(X, E)$ and extends to a mapping $\nabla_{\text {End }}$ on $\mathscr{C}^{0, \bullet}(X, \operatorname{End} E)$ and $\nabla_{\text {End }}^{2}=0$. If

$$
u=\frac{\sigma}{\nabla_{\mathrm{End}} \sigma}=\sigma+\sigma(\bar{\partial} \sigma)+\sigma(\bar{\partial} \sigma)^{2}+\cdots
$$

it turns out that $\nabla_{\mathrm{End}} u=I_{E}$ in $X \backslash Z$. One can define a canonical current extension $U$ of $u$ across $Z$ as the analytic continuation to $\lambda=0$ of $|F|^{2 \lambda} u$, where $F$ is any holomorphic function that vanishes on $Z$. In the same way we can define the current $R=\left.\bar{\partial}|F|^{2 \lambda} \wedge u\right|_{\lambda=0}$ with support on $Z$, and then

$$
\nabla_{\text {End }} U=I_{E}-R \text {. }
$$

More precisely

$$
R=\sum_{\ell} R^{\ell}=\sum_{\ell k} R_{k}^{\ell},
$$

where $R_{k}^{\ell}$ is a $(0, k-\ell)$-current that takes values in $\operatorname{Hom}\left(E_{\ell}, E_{k}\right)$, i.e.,

$$
R_{k}^{\ell} \in \mathscr{C}^{0, k-\ell}\left(X, \operatorname{Hom}\left(E_{\ell}, E_{k}\right)\right) .
$$

It is shown in [5] that the currents $U$ and $R$ both are pseudomeromorphic. Moreover we have (Proposition 2.2 in [4]) 
Proposition 2.3. - If $\phi \in \theta\left(E_{\ell}\right)$ and $f_{\ell} \phi=R^{\ell} \phi=0$, then $\phi=f_{\ell+1} \psi$ has local solutions $\psi \in \Theta\left(E_{\ell+1}\right)$. If $R^{\ell+1}=0$, then $\phi=f_{\ell+1} \psi$ has local holomorphic solutions $\psi$ if and only if $f_{\ell} \phi=R^{\ell} \phi=0$.

Since (2.3) is generically exact, so is its dual complex

$$
0 \rightarrow E_{-M}^{*} \stackrel{f_{-M+1}^{*}}{\longrightarrow} \cdots \stackrel{f_{N}^{*}}{\longrightarrow} E_{N}^{*} \rightarrow 0
$$

of Hermitian vector bundles, and we have the corresponding dual complex of locally free sheaves

$$
0 \rightarrow \theta\left(E_{-M}^{*}\right) \stackrel{f_{-M+1}^{*}}{\longrightarrow} \cdots \stackrel{f_{N}^{*}}{\longrightarrow} \vartheta\left(E_{N}^{*}\right) \rightarrow 0 .
$$

Using the induced metrics, we get a residue current

$$
R^{*}=\sum_{k}\left(R^{*}\right)^{k}=\sum_{k, \ell}\left(R^{*}\right)_{\ell}^{k}
$$

where $\left(R^{*}\right)_{\ell}^{k}$ takes values in $\operatorname{Hom}\left(E_{k}^{*}, E_{\ell}^{*}\right)$.

Proposition 2.4. - Using the natural isomorphisms $\operatorname{Hom}\left(E_{k}^{*}, E_{\ell}^{*}\right)=$ $\operatorname{Hom}\left(E_{\ell}, E_{k}\right)$ we have that $\left(R^{*}\right)_{\ell}^{k}=R_{k}^{\ell}$.

Proof. - It is readily verified that the adjoint $\sigma^{*}: E^{*} \rightarrow E^{*}$ of $\sigma: E \rightarrow E$ over $X \backslash Z$ is the minimal inverse of $f^{*}$. Therefore,

$$
u^{*}=\left(\sigma+\sigma(\bar{\partial} \sigma)+\sigma(\bar{\partial} \sigma)^{2}+\cdots\right)^{*}=\sigma^{*}+\sigma^{*}\left(\bar{\partial} \sigma^{*}\right)+\sigma^{*}\left(\bar{\partial} \sigma^{*}\right)^{2}+\cdots,
$$

since, see [4], $\sigma^{*} \bar{\partial} \sigma^{*}=\left(\bar{\partial} \sigma^{*}\right) \sigma^{*}$. Now the proposition follows.

If $\xi \in \Theta\left(E_{k}^{*}\right)$ and $\phi \in \Theta\left(E_{\ell}\right)$ we write

$$
\xi R_{k}^{\ell} \phi=\phi\left(R^{*}\right)_{\ell}^{k} \xi .
$$

2.3. The injectivity of the analytic sheaf $\mathscr{C}$. - Here is a proof of Malgrange's theorem by residue calculus. Let $\mathcal{F}$ be any module over the local ring $\theta_{0}$ and let (1.1) be a resolution of $\mathcal{F}$. We have to prove that then the complex

$$
0 \rightarrow \operatorname{Hom}\left(\theta_{0}\left(E_{0}\right), \mathscr{C}\right) \stackrel{f_{1}^{*}}{\longrightarrow} \operatorname{Hom}\left(\theta_{0}\left(E_{1}\right), \mathscr{C}\right) \stackrel{f_{2}^{*}}{\longrightarrow}
$$

is exact except at $k=0$. Fix a natural number $N$. Given a smooth function $\phi$ in $X \subset \mathbb{C}^{n}$, let $\tilde{\phi}$ be the function

$$
\tilde{\phi}(\zeta, \omega)=\sum_{|\alpha|<N} \partial_{\bar{\zeta}}^{\alpha} \phi(\zeta)(\omega-\bar{\zeta})^{\alpha} / \alpha !
$$

in $\tilde{X}=\left\{(\zeta, \bar{\zeta}) \in \mathbb{C}^{2 n} ; \zeta \in X\right\}$. Then

$$
\tilde{\phi}(\zeta, \bar{\zeta})=\phi(\zeta), \quad \bar{\partial} \tilde{\phi}=O\left(|\omega-\bar{\zeta}|^{N}\right) .
$$

TOME $139-2011-\mathrm{N}^{\mathrm{O}} 4$ 
Moreover, if $f$ is holomorphic then $\widetilde{f \phi}=f \tilde{\phi}$. Combining the formulas in [2] with the construction in [1], we get

$$
\tilde{\phi}(z, \bar{z})=\int_{\zeta, \omega}\left(f_{k+1}(z) H^{k} U^{k}+H^{k} R^{k}+H^{k} U^{k-1} f_{k}\right) \wedge\left(\tilde{\phi}+\bar{\partial} \tilde{\phi} \wedge v^{z}\right) \wedge g,
$$

where $g$ is a suitable form in $\mathbb{C}^{2 n}$ with compact support and $v^{z}$ is the BochnerMartinelli form in $\mathbb{C}^{2 n}$ with pole at $(z, \bar{z})$, and $H^{\ell}$ are holomorphic forms. Since $R^{k}=0$ for $k \geq 1$ when (1.1) is a resolution, see Theorem 3.1 in [4], we have the homotopy formula

$$
\phi=f_{k+1} T_{k+1} \phi+T_{k}\left(f_{k} \phi\right), \quad k \geq 1,
$$

where

$$
T_{k} \phi(z)=\int_{\zeta, \omega} H^{k} U\left(\tilde{\phi}+\bar{\partial} \tilde{\phi} \wedge v^{z}\right) \wedge g^{z} .
$$

Moreover, as in [1] one can verify that $T_{k} \phi$ is of class $C^{M}$ if $N$ is large enough. If now $\mu$ has order at most $M$, then we have

$$
\mu=T_{k+1}^{*} f_{k+1}^{*} \mu+f_{k}^{*} T_{k}^{*} \mu
$$

so if $f_{k+1}^{*} \mu=0$, then $\mu=f_{k}^{*} \gamma$ if $\gamma=T_{k}^{*} \mu$. Thus (2.8) is exact at $k$.

\section{Proofs of the main results}

Assume that $\mathcal{F}$ is a coherent sheaf of positive codimension $p$, and let (1.1) be a (locally) free resolution of $\mathcal{F}=\mathscr{\theta}\left(E_{0}\right) / \mathcal{I}$. Moreover, assume that $f_{1}$ is generically surjective so that the corresponding vector bundle complex

$$
0 \rightarrow E_{N} \stackrel{f_{N}}{\longrightarrow} \cdots \stackrel{f_{3}}{\longrightarrow} E_{2} \stackrel{f_{2}}{\longrightarrow} E_{1} \stackrel{f_{1}}{\longrightarrow} E_{0} \rightarrow 0
$$

is generically exact. It follows from Proposition 2.1 that

$$
R^{0}=R_{p}^{0}+R_{p+1}^{0}+\cdots
$$

By Theorem 3.1 in [4], $R_{k}^{\ell}=0$ for each $\ell \geq 1$, i.e., $R=R^{0}$, and combining with Proposition 2.3 above we find that a $\phi \in \Theta\left(E_{0}\right)$ is in $\mathscr{I}$ if and only if $R \phi=0$. It is proved in Section 5 of [5] that $\mathcal{F}$ has pure codimension $p$ if and only if ann $R=\operatorname{ann} R_{p}$, i.e., Theorem 1.1 holds.

Proof of Theorem 1.2. - It follows from (2.5) that

$$
\bar{\partial} R_{k}=f_{k+1} R_{k+1}
$$

for each $k$. If $\xi \in \theta\left(E_{k}^{*}\right)$ and $f_{k+1}^{*} \xi=0$ we therefore have

$$
\bar{\partial}\left(\xi R_{k}\right)= \pm \xi \bar{\partial} R_{k}= \pm \xi f_{k+1} R_{k+1}= \pm\left(f_{k+1}^{*} \xi\right) R_{k+1}=0 .
$$


Thus $\xi R_{p}$ is $\bar{\partial}$-closed and since it is also pseudomeromorphic, cf., Proposition 2.1, it is in $\mathscr{C H} \mathcal{H}_{Z}$. Moreover, if $\xi=f_{p}^{*} \eta$, then

$$
\xi R_{p}=\left(f_{p}^{*} \eta\right) R_{p}=\eta f_{p} R_{p}=\eta \bar{\partial} R_{p-1}=0
$$

since $R_{k}=0$ for $k<p$. Thus $\xi R_{p}$ only depends on the cohomology class of $\xi$ in $H^{p}\left(\theta\left(E_{\bullet}^{*}\right)\right)$. We now choose another Hermitian metric on $E$ and let $\tilde{R}$ denote the current associated with the new metric. It is showed in [4] (see the proof of Theorem 4.4) that then

$$
R_{p}-\tilde{R}_{p}=f_{p+1} M_{p+1}^{0}
$$

for a certain residue current $M$. Thus $\xi R_{p}=\xi \tilde{R}_{p}$. It follows that the mapping (1.4) is well-defined and independent of the Hermitian metric on $E$.

It is enough to prove the invariance at a fixed point $x$, so we consider stalks of the sheaves at $x$. It is well-known that then our resolution $\theta_{x}\left(E_{\bullet}\right), f_{\bullet}$ can be written

$$
\theta_{x}\left(E_{\bullet}^{\prime} \oplus E_{\bullet}^{\prime \prime}\right) \simeq \theta_{x}\left(E_{\bullet}^{\prime}\right) \oplus \theta_{x}\left(E_{\bullet}^{\prime \prime}\right), f_{\bullet}=f_{\bullet}^{\prime} \oplus f_{\bullet}^{\prime \prime},
$$

where $\theta_{x}\left(E_{\bullet}^{\prime}\right)$ is a resolution of $\mathcal{F}_{x}$ and (since we assume that $E_{0}$ has minimal rank) $\theta_{x}\left(E_{k}^{\prime \prime}\right), k \geq 1$, is a resolution of $\vartheta_{x}\left(E_{0}^{\prime \prime}\right)=0$. It follows that the natural mapping $H^{p}\left(\theta_{x}\left(\left(E_{\bullet}^{\prime}\right)^{*}\right) \rightarrow H^{p}\left(\theta_{x}\left(\left(E_{\bullet}\right)^{*}\right)\right), \xi^{\prime} \mapsto\left(\xi^{\prime}, 0\right)\right.$, is an isomorphism. Moreover, if we choose a metric on $E_{k}=E_{k}^{\prime} \oplus E_{k}^{\prime \prime}$ that respects the direct sum, then the resulting current $R$ is $R^{\prime} \oplus 0$, where $R^{\prime}$ is the current associated with $\theta_{x}\left(E_{\bullet}^{\prime}\right)$. Since all minimal resolutions are isomorphic, the mapping (1.5) is therefore well-defined.

It remains to check that (1.5) is non-degenerate. If $\xi \in \Theta\left(E_{p}^{*}\right)$ with $f_{p+1}^{*} \xi=0$ and $\xi R_{p} \phi=0$ for all $\phi \in \Theta\left(E_{0}\right)$, then clearly $\xi R_{p}=0$. Since $R=R_{p}^{0}$, by Proposition 2.4 therefore $\left(R^{*}\right)_{\ell}^{p} \xi=0$ for all $\ell$, and now it follows from Proposition 2.3 that $\xi=f_{p}^{*} \eta$ for some $\eta$. Thus (the class of) $\xi$ is zero in $\mathcal{H}^{p}\left(\theta\left(E_{\bullet}^{*}\right)\right)$.

Now, assume that $\xi R_{p} \phi=0$ for all $\xi$ such that $f_{p+1}^{*} \xi=0$. If $\mathcal{F}$ is CohenMacaulay and $N=p$, then $f_{p+1}^{*}=0$ so the assumption implies that $R_{p} \phi=0$, and thus $\phi \in \mathcal{I}$. However, generically on $Z, \mathcal{F}$ is Cohen-Macaulay, and hence for an arbitrary resolution we must have that $R_{p} \phi=0$ outside a variety of codimension $\geq p+1$. Since $R_{p} \phi$ is pseudomeromorphic with bidegree $(0, p)$ it follows from Proposition 2.1 that $R_{p} \phi$ vanishes identically. If we in addition assume that $\mathcal{F}$ has pure codimension it follows from Theorem 1.1 that $\phi \in \mathcal{I}$. Thus the pairing is non-degenerate.

EXAmple 4 (The Cohen-Macaulay case). - It is well-known, see, e.g., [12], that $\mathcal{F}$ is Cohen-Macaulay if and only if it admits resolutions of length $p=$ $\operatorname{codim} Z$. If (1.1) is a resolution with $N=p$, then $R=R_{p}^{0}$, and hence $R^{*}=$ $\left(R^{*}\right)_{p}^{0}$. It follows from Proposition 2.3, applied to $R^{*}$, that the dual complex 
(1.2) is a resolution of $\Theta\left(E_{0}^{*}\right) / \mathcal{I}^{*}$ and, in particular, that $\Theta\left(E_{0}^{*}\right) / \mathcal{I}^{*}$ is CohenMacaulay.

Proof of Theorem 1.5. - Let

$$
\mathscr{L}^{\nu}=\sum_{\ell+k=\nu} \mathscr{C}^{0, k}\left(E_{\ell}^{*}\right)
$$

be the total complex with differential $\nabla^{*}=f^{*}-\bar{\partial}$ associated with the double complex (1.8). We then have natural isomorphisms

$$
\mathcal{H}^{k}\left(\Theta\left(E_{\bullet}^{*}\right)\right) \simeq \mathcal{H}^{k}(\mathscr{L})=_{\text {def }} \frac{\operatorname{Ker}_{\nabla^{*}} \mathscr{L}^{k}}{\nabla^{*} \mathscr{L}^{k-1}} \simeq \mathcal{H}^{k}\left(\operatorname{Ham}\left(\mathcal{F}, \mathscr{C}^{0, \bullet}\right)\right) .
$$

The naturality means that the ismorphisms are induced by the natural mappings $\Theta\left(E_{k}^{*}\right) \rightarrow \mathcal{L}^{k}$ and $\operatorname{Ham}\left(\mathcal{F}, \mathscr{C}^{0, \ell}\right) \rightarrow \mathcal{L}^{k}$, respectively, and that $\xi \in \Theta\left(E_{k}^{*}\right)$ such that $f_{k+1}^{*} \xi=0$ defines the same class as $\mu \in \operatorname{Hom}\left(\mathcal{F}, \mathscr{C}^{0, k}\right)$ with $\bar{\partial} \mu=0$ if and only if there is $W \in \mathscr{L}^{k-1}$ such that $\nabla^{*} W=\xi-\mu$.

If now $\xi \in \Theta\left(E_{k}^{*}\right)$ and $f_{k+1}^{*} \xi=0$, then $\nabla^{*} \xi=0$, and hence

$$
\nabla^{*}\left(U^{*}\right)^{k} \xi=\xi-\left(R^{*}\right)^{k} \xi=\xi-\xi R_{k}
$$

cf., (2.5) and Proposition 2.4 above. Therefore the composed mapping in (1.10) coincides with the isomorphisms in (1.9). It is readily verified that the second mapping in (1.10) is injective, see, e.g., Lemma 3.3 in [3], and hence both mappings must be isomorphisms. Thus Theorem 1.5 is proved.

We think it may be enlightening with a proof of the first isomorphism in (1.10) that does not rely on Malgrange's theorem. We already know from Theorem 1.2 that this mapping is injective, so we have to prove the surjectivity. The proof is based on the following lemma.

LEMMA 3.1. - If there is a current $W \in \mathscr{L}^{p-1}$ such that $\nabla^{*} W=\mu \in$ CHC ${ }_{Z}\left(E_{0}^{*}\right)$, then $\mu=0$.

Proof. - Let $u$ be a smooth form $u$ such that $\nabla_{\text {End }}^{*} u=I_{E^{*}}$ in $X \backslash Z$. For a given neighborhood $\omega$ of $Z$, take a cutoff function $\chi$ with support in $\omega$ and equal to 1 in some neighborhood of $Z$. Then $g=\chi I_{E^{*}}-\bar{\partial} \chi \wedge u$ is smooth with compact support in $\omega$, equal to $I_{E^{*}}$ in a neighborhood of $Z$, and moreover $\nabla^{*} g=0$. Therefore, $\nabla^{*}(g W)=g \mu=\mu$ and hence, for degree reasons, we have a solution $\bar{\partial} w=\mu$ with support in $\omega$. Since $\omega \supset Z$ is arbitrary it follows, cf., Lemma 3.3 in [3], that $\mu=0$.

Since (1.8) is exact in $k$ except at $k=0$, the first equivalence in (3.3) holds. Take $\mu \in \operatorname{Ham}\left(\mathcal{F}, \mathscr{C H}_{Z}\right)$. Then $\nabla^{*} \mu=\left(f_{1}^{*}-\bar{\partial}\right) \mu=0$ so by (3.3) (with $k=p$ ) there is $\xi \in \Theta\left(E_{p}^{*}\right)$ such that $\nabla^{*} W=\xi-\mu$ has a current solution $W \in \mathcal{L}^{p-1}$. In view of (3.4) it now follows from Lemma 3.1 that $\mu=\xi R_{p}^{0}$. 


\section{An algebraic approach}

In this section we indicate how Theorem 1.2 and its corollaries (except for the concrete representation $\xi \mapsto \xi R_{p}$ ) can be proved algebraically. This material has been communicated to us by Jan-Erik Björk. Whereas our residue proof above was based on Theorem 1.1, the algebraic proof instead relies on the following fundamental result due to J-E Roos, [18]:

THEOREM 4.1. - The sheaf $\mathcal{F}$ has pure codimension $p$ if and only if the natural mapping

$$
\mathcal{F} \rightarrow \mathcal{E} x t^{p}\left(\mathcal{E} x t^{p}(\mathcal{F}, \Theta), \Theta\right)
$$

is injective.

Assume that

$$
0 \rightarrow \mathscr{I} \rightarrow \mathcal{\Theta}\left(E_{0}\right) \rightarrow \mathcal{F} \rightarrow 0
$$

is exact as before. Moreover, let us assume to begin with that we already know the isomorphisms (1.10). In particular we then have that

$$
\mathcal{E} x t^{p}(\mathcal{F}, \theta) \simeq \mathcal{H} \operatorname{Cam}\left(\mathcal{F}, \mathscr{C H}_{Z}\right) .
$$

Thus we can choose (locally) a finite number of generators $\mu_{\alpha}, \alpha \in A$, for $\operatorname{Ham}\left(\mathcal{F}, \mathscr{C H} \mathcal{H}_{Z}\right)$ and get an exact sequence

$$
0 \rightarrow \unlhd \rightarrow \theta^{A} \rightarrow \operatorname{Hom}\left(\mathcal{F}, \mathscr{H}_{Z}\right) \rightarrow 0
$$

and therefore we have, with $\mathcal{M}=\mathscr{H} \operatorname{Cam}\left(\mathcal{F}, \mathscr{H} \mathcal{H}_{Z}\right) \simeq \mathcal{E} x t^{p}(\mathcal{F}, \Theta)$, that

$$
\mathcal{E x t}(\mathcal{M}, \theta) \simeq \operatorname{Ham}\left(\mathcal{M}, \mathscr{C H} \mathcal{H}_{Z}\right) .
$$

We claim that the canonical mapping (4.1) is given by

$$
\vartheta\left(E_{0}\right) \ni \phi \mapsto\left(\mu_{\alpha} \phi\right)_{\alpha} .
$$

In fact, clearly it is a mapping from $\mathcal{F}=\mathscr{\theta}\left(E_{0}\right) / \mathcal{I}$, since each $\mu_{\alpha}$ is. Moreover, if $\left(\psi_{\alpha}\right) \in \mathcal{I}$, then by definition $\sum_{\alpha} \psi_{\alpha} \mu_{\alpha}=0$, and hence $\left(\mu_{\alpha} \phi\right)_{\alpha}$ defines an element in

$$
\operatorname{Ham}\left(\theta^{A} / \mathcal{I}, \mathscr{G} \mathcal{H}_{Z}\right) \simeq \varepsilon x t^{p}\left(\varepsilon x t^{p}(\mathcal{F}, \theta), \theta\right) .
$$

One can verify that this mapping is independent of the choice of generators, and must be the canonical mapping (4.1).

It follows that (4.1) is injective if and only if the equality (1.6) holds. If $\mathcal{F}$ has pure codimension $p$, by Theorem 4.1 therefore (1.6) holds and then Corollary 1.3 as well as Theorem 1.2 follow.

TOME $139-2011-\mathrm{N}^{\mathrm{O}} 4$ 
REMARK 3. - We actually get a residue proof of Theorem 4.1: If $\mathcal{F}$ has pure codimension $p$ we know that (1.6) holds by the residue theory, and thus (4.1) is injective. On the other hand, it is not hard to see, e.g., it follows from [5], that the annihilator of (a set of) currents in $\mathscr{C H} \mathcal{H}_{Z}$ must have pure codimension $p$. Thus the injectivity of (4.1) implies that $\mathcal{F}$ has pure codimension.

Let us conclude with a brief discussion of (1.10). The Dickenstein-Sessa decomposition (1.12) is well-known; see [10] in case of a complete intersection and [7] for the general case. Malgrange also proved that $\mathscr{C}_{Z}$ is stalkwise injective as an analytic sheaf. Using these two facts and considering the spectral sequence obtained from the double complex

$$
\operatorname{Ham}\left(\theta\left(E_{\ell}\right), \mathscr{C}_{Z}^{0, k}\right)
$$

one can conclude that the second mapping in (1.10) is indeed an isomorphism, and hence both of them. However, we omit the details.

\section{The absolute case and Bezoutians}

Let $I \subset \theta_{0}\left(E_{0}\right)$ be a submodule of the free module $\theta_{0}\left(E_{0}\right)$ over the local ring $\theta_{0}$ such that the zero variety of ann $\left(\theta_{0}\left(E_{0}\right) / I\right)$ is $Z=\{0\}$. Moreover, let (1.1) be a resolution of $\theta_{0}\left(E_{0}\right) / I$ of length $n$. From Corollary 1.4 we have the non-degenerate pairing

$$
\vartheta_{0}\left(E_{0}\right) / I \times \vartheta_{0}\left(E_{n}^{*}\right) / I^{*} \rightarrow \mathscr{H} \epsilon_{\{0\}} .
$$

Let $\alpha \in \Omega_{0}^{n}$ be a germ of a nonvanishing holomorphic $(n, 0)$-form at the origin, and let

$$
\mathscr{C} \mathcal{H}_{\{0\}} \rightarrow \mathbb{C}, \quad \mu \rightarrow \mu . \alpha=\int \alpha \wedge \mu .
$$

Then we have

Proposition 5.1. - The composed mapping

$$
\vartheta_{0}\left(E_{0}\right) / I \times \vartheta_{0}\left(E_{n}^{*}\right) / I^{*} \rightarrow \mathbb{C}
$$

is a non-degenerate pairing.

Proof. - If $\phi \in \theta_{0}\left(E_{0}\right)$ is not in $I$, then there is some $\xi \in \theta_{0}\left(E_{n}^{*}\right)$ such that $\mu=\xi R_{n} \phi$ is not identically zero. Since $\mu$ is in $\mathscr{C} \mathcal{H}_{\{0\}}$ there is some holomorphic $\psi \in \theta_{0}$ such that $\psi \mu \neq 0$. Thus $\psi \xi R_{n} \phi . \alpha=\psi \mu . \alpha \neq 0$. Since we can interchange the roles of $I$ and $I^{*}$ the proposition follows. 
In particular, we obtain an (non-canonical) isomorphism

$$
\left(\theta_{0}\left(E_{0}\right) / I\right)^{*} \simeq \theta_{0}\left(E_{n}^{*}\right) / I^{*} .
$$

Notice that the form $u_{n}^{0}$ defines a $\operatorname{Hom}\left(E_{0}, E_{n}\right)$-valued Dolbeault cohomology class $\omega$ in $\mathcal{U} \backslash\{0\}$. Since $\bar{\partial} U_{n}^{0}=R_{n}$ we have

$$
(\xi, \phi)=\int_{|\zeta|=\epsilon} \alpha \wedge \xi \omega \phi
$$

From (5.4) in [4] we get the representation

$$
\phi(z)=f_{1}(z) \int H^{1} U \phi \wedge g+\int H_{n}^{0} R_{n} \phi \wedge g, \quad \phi \in \Theta\left(E_{0}\right) ;
$$

here $H^{1}$ is a holomorphic $\operatorname{Hom}\left(E, E_{1}\right)$-valued form, $H_{n}^{0}$ is a $\operatorname{Hom}\left(E_{n}, E_{0}\right)$ valued holomorphic $(n, 0)$-form, so that

$$
H_{n}^{0}=h_{n}^{0} \alpha,
$$

and $g$ is the form (5.2) in [4]. It has compact support and depends holomorphically on $z$. Moreover, $g=\chi+\cdots$, where the dots denote smooth forms of positive bidegree, so modulo $I$ we have

$$
\phi(z) \equiv_{\bmod I} \int_{|\zeta|=\epsilon} H_{n}^{0}(\cdot, z) \omega \phi,
$$

and hence

$$
\phi(z) \equiv_{\bmod I}\left(h_{n}^{0}(\cdot, z), \phi\right) .
$$

This means that we can consider $h_{n}^{0}$ as a (generalized) Bezoutian, cf., [8]. For each analytic functional $\mu$ on $\theta_{0}\left(E_{0}\right)$ that vanishes on $I$ there is a unique element $\xi$ in $\theta_{0}\left(E_{n}^{*}\right) / I^{*}$ such that the action on $\theta_{0}\left(E_{0}\right)$ modulo $I$ coincides with $\mu . \phi=(\xi, \phi)$ in view of (5.2). More explicitly we have

$$
\xi(\zeta)=\mu_{z}\left(h_{n}^{0}(\zeta, \cdot)\right) .
$$

In the classical case of a complete intersection $I=\left(f^{1}, \ldots, f^{n}\right)$, if we choose Hefer forms, i.e., $(1,0)$-forms $h_{k}=\sum_{j} h_{j k} d \zeta_{j}$ such that $\sum h_{j k}\left(\zeta_{j}-z_{j}\right)=$ $f^{k}(z)-f^{k}(\zeta)$, and $\alpha=d \zeta_{1} \wedge \cdots \wedge d \zeta_{n}$, then, cf., [2], it turns out that $h_{n}^{0}=$ $\operatorname{det}\left(h_{j k}\right)$; this is a well-known formula, cf., [8], for the Bezoutian in this case.

\section{Cohomological residues}

The Coleff-Herrera currents admit a nice intrinsic way of expressing the action of holomorphic differential operators, but the very definition relies on Hironaka's theorem about the existence of resolutions of singularities. In [10] and [17] there is also a cohomological way of expressing the duality for a complete intersection. We have the following cohomological version of Corollary 1.3. 
THEOREM 6.1. - With the assumptions and notation as in Corollary 1.3 and with $w_{j}=\xi_{j} u_{p}^{0}$ outside $Z$ we have for $\phi \in \Theta\left(E_{0}\right)$ that

$$
w_{j} \phi . \psi=0, \quad \psi \in \mathscr{D}, \quad \bar{\partial} \psi=0 \text { close to } Z,
$$

if and only if $\phi \in I$.

In fact, $w_{j}$ can be extended to $W_{j}=\xi_{j} U_{p}^{0}$ across $Z$ and

$$
\bar{\partial} W_{j}=\bar{\partial}\left(\xi_{j} U_{p}^{0}\right)= \pm \xi_{j} R_{p}= \pm \mu_{j} .
$$

One can now verify that (6.1) implies that $\mu_{j} \phi=0$, see, e.g., Theorem 6.1 in [3]. Thus Theorem 6.1 follows from Corollary 1.3. Moreover, (6.1) is equivalent to that $w_{j} \phi$ are $\bar{\partial}$-exact in $X \backslash Z$ for each Stein neighborhood $X$.

In the complete intersection case, as well as the Cohen-Macaulay case, see [14], the proof is algebraic and only involves "cohomological" residues, whereas the proof of Theorem 6.1 here is obtained via the residue calculus. It is reasonable to believe that one can produce a purely algebraic proof (thus avoiding Hironaka's theorem) based on Roos' theorem, cf., Section 4.

\section{Higher $E x t$ sheaves}

Assume that $\mathcal{F}=\mathscr{\theta}\left(E_{0}\right) / \mathcal{I}$ has codimension $p$ as before. In view of Proposition 2.1 and (1.10) one could guess that $\delta x t^{k}(\mathcal{F}, \theta)$ for $k>p$ could be represented by cohomology of pseudomeromorphic currents. We have the following partial result.

THEOREM 7.1. - Assume that (1.1) is a resolution of $\mathcal{F}$ and $R$ is the associated residue current (with respect to some given metric). For each $k$, $\vartheta\left(E_{k}^{*}\right) \ni \xi \mapsto \xi R_{k}$ induces an invariant injectice mapping

$$
\mathcal{E} x t^{k}(\mathcal{F}, \theta) \rightarrow \mathcal{H}^{k}\left(\mathcal{H} \operatorname{tam}\left(\mathcal{F}, \mathscr{P} M^{0, \bullet}\right)\right) .
$$

Moreover, the composed mapping

$$
\mathcal{H}^{k}\left(\Theta\left(E_{\bullet}^{*}\right)\right) \rightarrow \mathcal{H}^{k}\left(\mathcal{H} \operatorname{tam}\left(\mathcal{F}, \mathscr{P} M^{0, \bullet}\right)\right) \rightarrow \mathcal{H}^{k}\left(\mathcal{H} \operatorname{Com}\left(\mathcal{F}, \mathscr{C}^{0, \bullet}\right)\right)
$$

coincides with the natural isomorphism (1.9).

REMARK 4. - If we widen the definition of $\mathscr{P} M$ slightly so that it is preserved under any surjective holomorphic mapping rather than just modifications, then the $\bar{\partial}$-complex $\mathscr{P} M_{0, \bullet}$ is exact and thus it is a fine resolution of the sheaf $\mathscr{\theta}$. It is reasonable to believe that $\mathscr{P M}$ so defined is stalkwise injective. If this is true, by considering the double complex $\mathscr{P} M_{0, k}\left(E_{\ell}^{*}\right)$, we could conclude that the first mapping in (7.2) is an isomorphism for any $k$, and hence that both mappings are. 
Let $Z_{k}$ be the analytic set where the mapping $f_{k}$ in (1.1) does not have optimal rank. It is well-known and not hard to see that these sets are independent of the resolution and hence invariants of the sheaf $\mathcal{F}$. Then

$$
\cdots Z_{k+1} \subset Z_{k} \subset \cdots \subset Z_{p+1} \subset Z_{\text {sing }} \subset Z_{p}=Z
$$

and the Buchsbaum-Eisenbud theorem, see [12], states that the codimension of $Z_{k}$ is at least $k$. Moreover, by Corollary 20.14 in [12], $\mathcal{F}$ has pure codimension $p$ if and only if

$$
\operatorname{codim} Z_{k} \geq k+1, \quad k>p .
$$

Notice also that $\mathcal{F}$ is Cohen-Macaulay if and only if $Z_{k}=\varnothing$ for all $k>p$.

Proof of Theorem 1.1. - If $\mathcal{F}$ is pure, using (7.3), it follows as in (the proof of) Lemma 5.2 in [5], that ann $R_{p}=$ ann $R$, and thus ann $R_{p}=\mathscr{J}$. Conversely, assume that ann $R_{p}=\mathcal{I}$. It follows from Proposition 5.3 in [5] that ann $R_{p}$ must be an intersection of primary modules of codimension $p$, and hence $\mathscr{I}=\operatorname{ann} R_{p}$ is pure.

Proof of Theorem \%.1. - Since $R \phi=0$ for $\phi \in I$, it follows from (3.2) that the first mapping in (7.2) is well-defined, and in view of (3.4) the composed mapping coincides with the natural isomorphism. It follows that the first mapping must be injective. (This is also easily seen by a direct argument that avoids Malgrange's theorem: Assume that $\xi R_{k}=\bar{\partial} \gamma$ for some $\gamma$ in $\operatorname{Ham}\left(\mathcal{F}, \mathscr{P} M^{0, k-1}\right)$. Then $\xi R_{k}=\nabla^{*} \gamma$, so that $\nabla^{*}\left(U^{*} \xi-\gamma\right)=\xi$ and hence $\xi=0$ in $\mathcal{H}^{k}\left(\theta\left(E_{\bullet}^{*}\right)\right)$ in view of the first isomorphism in (3.3).)

If $\tilde{R}$ denotes the current associated with another metric, then as before there is a current $M$ with support on $Z$ such that

$$
\nabla_{\text {End }} M=R-\tilde{R}
$$

In fact, if

$$
u=\sigma / \nabla_{\text {End }} \sigma=\sigma+(\bar{\partial} \sigma) \sigma+(\bar{\partial} \sigma)^{2} \sigma+\cdots
$$

and $\tilde{u}$ is the analogous form corresponding to the new metric, then, cf., [4], we can take

$$
M=\left.\bar{\partial}|F|^{2 \lambda} \wedge u \tilde{u}\right|_{\lambda=0}
$$

Now, if $\xi \in \Theta\left(E_{k}^{*}\right)$ and $f_{k+1}^{*} \xi=0$, then

$$
\xi\left(R_{k}-\tilde{R}_{k}\right)=\xi\left(\nabla_{\text {End }} M\right)^{0}=\xi\left(f_{k+1} M_{k+1}^{0}-\bar{\partial} M_{k}^{0}\right)=- \pm \bar{\partial}\left(\xi M_{k}^{0}\right) .
$$


Thus we must show that $\xi M_{k}^{0} \phi=0$ for $\phi \in I$. If $\phi \in \mathcal{I}$, then $\phi=f_{1} \psi=\nabla \psi$ for some $\psi \in \theta\left(E_{1}\right)$. Thus

$$
\begin{aligned}
& \xi M_{k}^{0} \phi=\xi M(\nabla \psi)=\xi\left(\nabla_{\text {End }} M\right) \psi-\xi \nabla(M \psi)= \\
& \quad \xi\left(R_{k}^{1}-\tilde{R}_{k}^{1}\right) \psi-\xi f_{k+1} M_{k+1}^{1} \psi+\xi \bar{\partial} M_{k}^{1} \psi=\xi \bar{\partial} M_{k}^{1} \psi
\end{aligned}
$$

since $R^{1}=\tilde{R}^{1}=0$, so it is enough to check that $M_{k}^{1}=0$, which we prove by induction over $k$ : First notice that $M_{k}^{1}$ must vanish for $k \leq p+1$ since it has bidegree $(0, k-2)$ and has support on $Z$ that has codimension $p$. Now suppose that we have proved that $M_{k}^{1}=0$. Outside $Z_{k+1}$ the mapping $\sigma_{k+1}$ is smooth so we have, cf., (7.4) and the definition of $R$,

$$
M_{k+1}^{1}=\sigma_{k+1} \tilde{R}_{k+1}^{1}+\left(\bar{\partial} \sigma_{k+1}\right) M_{k}^{1}=\left(\bar{\partial} \sigma_{k+1}\right) M_{k}^{1}=0
$$

there since $\tilde{R}^{1}=0$. Thus $M_{k+1}^{1}$ has support on $Z_{k+1}$ and for degree reasons it must vanish identically.

Outside the set $Z_{k}$, there is a resolution (1.1) of $\mathcal{F}$ with $N<k$, and it follows that then $\mathcal{E} x t^{k}(\mathcal{F}, \theta) \simeq \mathcal{H}^{k}\left(\Theta\left(E_{\bullet}^{*}\right)\right)$ vanishes there; i.e., $\mathcal{E} x t^{k}(\mathcal{F}, \Theta)$ has its support on $Z_{k}$. On the other hand, if $\varepsilon x t^{\ell}(\mathcal{F}, \theta)=0$ for all $\ell \geq k$, then

$$
\theta\left(E_{k-1}^{*}\right) \stackrel{f_{k}^{*}}{\longrightarrow} \Theta\left(E_{k}^{*}\right) \stackrel{f_{k+1}^{*}}{\longrightarrow} \cdots \stackrel{f_{N}^{*}}{\longrightarrow} \Theta\left(E_{N}^{*}\right) \rightarrow 0
$$

is exact, and hence all the mappings must have constant rank, so we must be outside $Z_{k}$. It follows that

$$
\operatorname{supp} \varepsilon x t^{k}(\mathcal{F}, \theta) \subset Z_{k} \subset \bigcup_{\ell \geq k} \operatorname{supp} \delta x t^{\ell}(\mathcal{F}, \theta) \text {. }
$$

If $\mathcal{F}$ has pure codimension $p$, then it follows from (7.5) and Eisenbud's theorem mentioned above that the support of $\delta x t^{k}(\mathcal{F}, \theta)$ has at least codimension $k+1$; a fact that was already established by Roos in [18].

On the other hand, if we have not pure codimension somewhere, then for some $k>p, \operatorname{codim} Z_{k}$ has codimension $k$. It follows from (7.5) that then the support $V$ of $\mathcal{E} x t^{k}(\mathcal{F}, \theta)$ has codimension $k$ here $\left(\right.$ since $\operatorname{supp} \mathcal{E} x t^{\ell}(\mathcal{F}, \theta)$ for $\ell>k$ have higher codimension). By the coherence there is $\xi \in \theta\left(E_{k}^{*}\right)$ whose cohomology class is (generically) nonvanishing on $V$. By Theorem 7.1 then the current $\xi R_{k}$ represents the corresponding nonvanishing class in $\operatorname{Ham}\left(\mathcal{F}, \mathscr{C}^{0, \bullet}\right)$.

\section{BIBLIOGRAPHY}

[1] M. Andersson - "Ideals of smooth functions and residue currents", $J$. Funct. Anal. 212 (2004), p. 76-88. 
[2] , "Integral representation with weights. II. Division and interpolation", Math. Z. 254 (2006), p. 315-332.

[3] _ "Uniqueness and factorization of Coleff-Herrera currents", Ann. Fac. Sci. Toulouse Math. 18 (2009), p. 651-661.

[4] M. Andersson \& E. Wulcan - "Residue currents with prescribed annihilator ideals", Ann. Sci. École Norm. Sup. 40 (2007), p. 985-1007.

[5] _ , "Decomposition of residue currents", J. reine angew. Math. 638 (2010), p. 103-118.

[6] J.-E. BJÖRK - Rings of differential operators, North-Holland Mathematical Library, vol. 21, North-Holland Publishing Co., 1979.

[7] _ "Residues and $\mathscr{D}$-modules", in The legacy of Niels Henrik Abel, Springer, 2004, p. 605-651.

[8] E. Cattani \& A. Dickenstein - "Introduction to residues and resultants", in Solving polynomial equations, Algorithms Comput. Math., vol. 14, Springer, 2005, p. 1-61.

[9] N. R. ColefF \& M. E. HerrerA - Les courants résiduels associés à une forme méromorphe, Lecture Notes in Math., vol. 633, Springer, 1978.

[10] A. Dickenstein \& C. Sessa - "Canonical representatives in moderate cohomology", Invent. Math. 80 (1985), p. 417-434.

[11] L. EHREnPREIS - Fourier analysis in several complex variables, Pure and Applied Mathematics, vol. XVII, Wiley-Interscience, 1970.

[12] D. Eisenbud - Commutative algebra, Graduate Texts in Math., vol. 150, Springer, 1995.

[13] L. HÖRMANDER - An introduction to complex analysis in several variables, revised ed., North-Holland Publishing Co., 1973.

[14] J. LundqVist - "A local Grothendieck duality theorem for CohenMacaulay ideals", to appear in Math Scand.

[15] U. OBERst - "The construction of Noetherian operators", J. Algebra 222 (1999), p. 595-620.

[16] V. P. PAlamodov - Linear differential operators with constant coefficients, Die Grundl. Math. Wiss., vol. 168, Springer, 1970.

[17] M. PASSARE - "Residues, currents, and their relation to ideals of holomorphic functions", Math. Scand. 62 (1988), p. 75-152.

[18] J.-E. Roos - "Bidualité et structure des foncteurs dérivés de lim dans la catégorie des modules sur un anneau régulier", C. R. Acad. Sci. Paris 254 (1962), p. 1720-1722. 